\title{
Do Needleless Knots have Similar Strength as the Krackow Suture? An In Vitro Porcine Tendon Study
}

\author{
Chih-Kai Hong MD, Ting-Hsuan Kuo, Ming-Long Yeh PhD, I-Ming Jou MD, PhD, \\ Cheng-Li Lin MD, Wei-Ren Su MD, MSc
}

Received: 6 July 2016/ Accepted: 28 October 2016/Published online: 7 November 2016

(C) The Association of Bone and Joint Surgeons (B) 2016

\begin{abstract}
Background Numerous needleless techniques for tendon graft fixation that feature several advantages have been reported. However, there are few studies that have compared the holding strength between the needleless techniques (rolling hitch and modified rolling hitch) and traditional suture methods.

Questions/purposes To compare the tendon graft-holding strength of the rolling hitch and modified rolling hitch with the Krackow stitch in an in vitro porcine biomechanical model.

Methods Thirty fresh-frozen porcine flexor profundus tendons were randomly divided into three groups of 10
\end{abstract}

Each author certifies that he or she, or a member of his or her immediate family, has no funding or commercial associations (eg, consultancies, stock ownership, equity interest, patent/licensing arrangements, etc) that might pose a conflict of interest in connection with the submitted article.

All ICMJE Conflict of Interest Forms for authors and Clinical Orthopaedics and Related Research ${ }^{\circledR}$ editors and board members are on file with the publication and can be viewed on request.

Clinical Orthopaedics and Related Research ${ }^{\mathbb{B}}$ neither advocates nor endorses the use of any treatment, drug, or device. Readers are encouraged to always seek additional information, including FDAapproval status, of any drug or device prior to clinical use.

This work was performed at the Department of Orthopaedic Surgery, National Cheng Kung University Hospital, College of Medicine, National Cheng Kung University, Tainan, Taiwan.

C.-K. Hong, I.-M. Jou, C.-L. Lin, W.-R. Su ( $ه)$ Department of Orthopaedic Surgery, National Cheng Kung University Hospital, College of Medicine, National Cheng Kung University, No. 138, Sheng-Li Road, Tainan 70428, Taiwan e-mail: suwr@ms28.hinet.net

T.-H. Kuo

Department of Medicine, National Cheng Kung University

College of Medicine, Tainan, Taiwan specimens. The experimental procedure was designed to assess elongation of the suture-tendon construct across the needleless tendon-grasping techniques and the Krackow stitch. All suture configurations were completed with a braided nonabsorbable suture. Each tendon was pretensioned to $100 \mathrm{~N}$ for three cycles, cyclically loaded from 50 to $200 \mathrm{~N}$ for 200 cycles, and then finally loaded to failure. Elongation, load to failure, and mode of failure were recorded for each specimen.

Results Five of the 10 rolling hitch specimens failed during cyclic loading. With the numbers available, elongation after cyclic loading was not different among the successful rolling hitch specimens $(19 \%[1.19 \mathrm{~cm} / 6.17 \mathrm{~cm}]$ $\pm 6 \%)$, modified rolling hitch fixations $(19 \%[1.11 \mathrm{~cm} /$ $5.93 \mathrm{~cm}] \pm 6 \%$ ), and Krackow stitch fixations (26\% [1.41 $\mathrm{cm} / 5.43 \mathrm{~cm}] \pm 6 \%$ ); ultimate failure loads also were not different among the rolling hitch fixations $(316 \pm 35 \mathrm{~N})$, modified rolling hitch fixations ( $342 \pm 14 \mathrm{~N})$, and Krackow stitches $(327 \pm 33 \mathrm{~N})$ with the numbers available.

Conclusions With the numbers available, the rolling hitch, modified rolling hitch, and Krackow stitch techniques were not different in terms of elongation after cyclic loading and to failure in this in vitro biomechanical evaluation.

Clinical Relevance Based on the biomechanical properties from this in vitro animal study, the modified rolling

\section{M.-L. Yeh}

Department of Biomedical Engineering, National Cheng Kung

University, Tainan, Taiwan

M.-L. Yeh, I.-M. Jou, C.-L. Lin, W.-R. Su

Medical Device Innovation Center, National Cheng Kung

University, Tainan, Taiwan 
hitch may be an attractive alternative for tendon graft fixation in ligament-reconstruction surgery. Future studies might consider further evaluating these needleless techniques in a cadaver model, in an in vivo animal model, and in an intraarticular model in which the testing is performed in the presence of synovial fluid.

\section{Introduction}

A reliable soft tissue suture technique is essential for ligament-reconstruction surgery, because adequate fixation allows for rehabilitation programs before completion of biologic fixation in the graft tunnel [1, 3, 6, 13, 15]. The whipstitch is a common suture technique for preparing and fixing tendon grafts [14]. Krackow et al. [11] proposed an alternative locking suture in 1986, which became a common technique for preparing a suture-tendon graft.

Several needleless grasping sutures, namely the modified Prusik knot, Wittstein suture loop, rolling hitch, modified finger-trap, and modified rolling hitch, have been proposed in recent years owing to inherent disadvantages of the previous techniques, including tendon damage caused by passing a needle, time consumption, and the potential risk of needlestick injury [7, 8, 12, 15, 17]. These needleless techniques have been shown to reduce cost, decrease exposure to needles, and shorten surgical time $[7,8,10,12,17]$.

Several studies have evaluated the biomechanical properties of the needleless suture techniques [2, 10, 12, 15]. Krappinger et al. [12] compared the
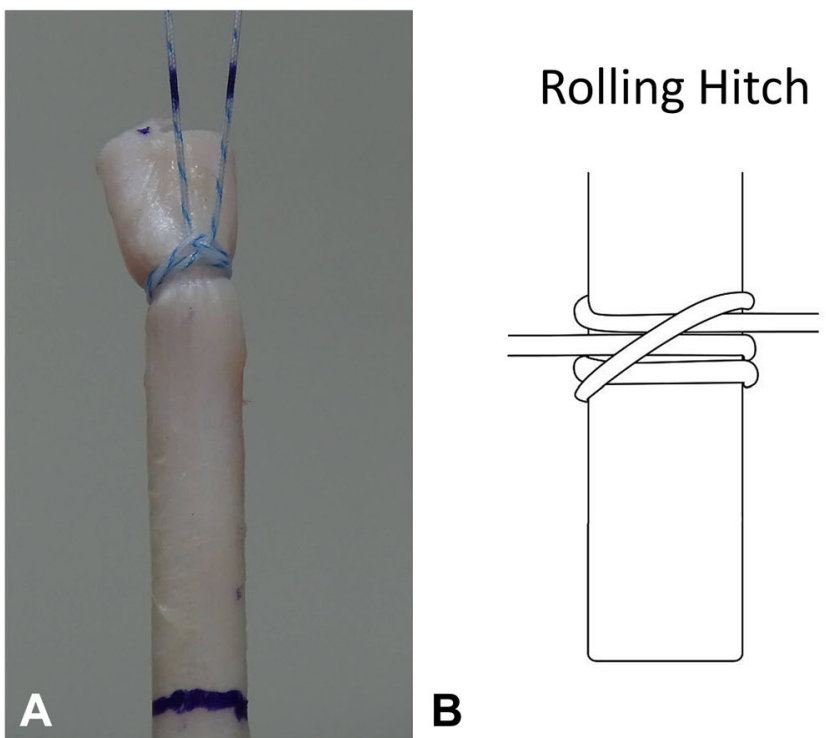

Fig. 1A-D (A) A photograph and (B) diagram of the rolling hitch, and (C) a photograph and (D) diagram of the modified rolling hitch are shown. (Illustrations $\mathrm{C}$ and $\mathrm{D}$ are reprinted with permission from Elsevier from Hong CK, Yeh ML, Jou IM, Lin CL, Chang CH, Su modified Prusik knot with the whipstitch, and found that the modified Prusik knot had similar mechanical properties as the whipstitch technique. Su et al. [15] proposed the modified finger-trap for securing tendon grafts, and showed that the modified finger-trap provided better biomechanical properties than the Krackow stitch. Hong et al. [10] evaluated the tendon graft holding strength of the modified Prusik knot, Wittstein suture loop, and modified rolling hitch; their results showed similar elongation after cyclic loading and loading to failure. Camarda et al. [2] further characterized the biomechanical effect of the modified rolling hitch and modified finger-trap, the results of which showed that the modified rolling hitch resulted in less elongation during cyclic loading and higher stiffness than did the modified finger-trap.

Although previous studies have suggested the modified rolling hitch to be an attractive alternative $[2,10]$, to our knowledge, no biomechanical study has simultaneously compared the holding strength of the modified rolling hitch or rolling hitch with the commonly used whipstitch. The aim of this study was to compare the biomechanical properties of the rolling hitch, modified rolling hitch, and Krackow stitch fixation. We hypothesized that there would be no differences in elongation and load to failure among these needleless suture techniques and the Krackow stitch.

\section{Materials and Methods}

Two needleless tendon-grasping techniques were investigated, namely the rolling hitch and modified rolling hitch

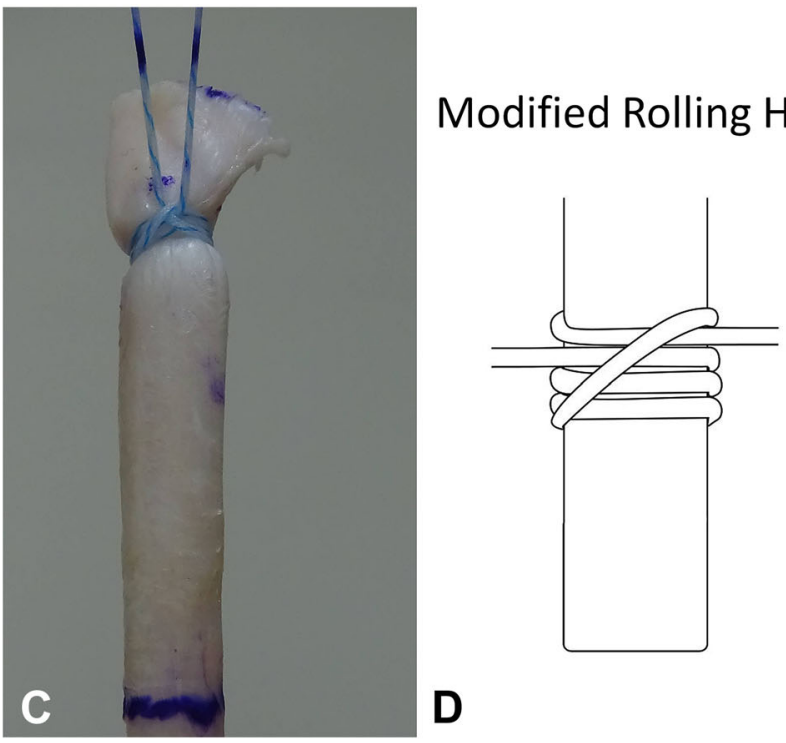

WR. Evaluation of 3 needleless grasping suture techniques for softtissue graft fixation: a porcine biomechanical study. Arthroscopy. 2015;31:1151-1155.) 
(Fig. 1). The Krackow stitch [11], performed with five locking loops along each side of the tendon, was set as the control group. A Number 2 FiberWire ${ }^{\circledR}$ suture (Arthrex, Naples, FL, USA) was used for each suture-tendon construct.

The rolling hitch starts by wrapping the suture around the tendon followed by making a second wrap; the working limb of the suture then is crossed over the other limb, and the procedure is completed with a half hitch by making a turn around the tendon and passing the working limb through it. The modified rolling hitch also starts by wrapping the suture around the tendon but is followed by making second and third wraps; a half hitch then is made by making a turn around the tendon and passing the working limb through it. All suture constructs were set at a 1-cm distance from the end of the tendon and performed by the same experienced orthopaedic surgeon (W-RS) for each specimen.

This study was granted an exemption from the institutional review board at the National Cheng Kung University Hospital, Tainan, Taiwan. Thirty fresh adult male porcine (mean age, 2 years) hindleg trotters stored at $-20^{\circ} \mathrm{C}$ were thawed to room temperature before testing. The entire flexor profundus tendon then was dissected, none of which appeared to have any degenerative or pathologic changes. The attached soft tissue, including the synovial sheath and vincula, were removed from the distal part of the tendons. The porcine tendons were kept moist by spraying with $0.9 \%$ saline solution during preparation and testing. A total of 30 tendons of equal length $(18 \mathrm{~cm})$ were obtained and randomly divided into three groups of 10 specimens each, with each group randomly assigned to receive one of the three suture configurations.

A transverse section 5 to $6 \mathrm{~mm}$ in thickness was taken from the distal end of each tendon before testing. Each section was photographed alongside a calibration scale. An 8.9-megapixel digital camera (EOS 60D; Canon ${ }^{\circledR}$, Tokyo, Japan) mounted on a tripod and image analysis software (SigmaScan Pro 5.0; Systat Software Inc, San Jose, CA, USA) were used to calculate the cross-sectional areas of the tendon sections. There were no differences in the crosssectional areas of the tendons among the rolling hitch (43 $\pm 5 \mathrm{~mm}^{2}$ ), modified rolling hitch $\left(42 \pm 4 \mathrm{~mm}^{2}\right.$ ), and Krackow stitch $\left(44 \pm 4 \mathrm{~mm}^{2}\right)(\mathrm{p}=0.426)$.

After each suture-tendon construct was completed, each specimen was mounted on a universal materials testing machine (AG-X; Shimadzu, Tokyo, Japan). A sinusoid mechanical clamp was used to fix the proximal end of the tendon, thereby allowing an equal length (9 $\mathrm{cm}$ ) of free tendon for testing (Fig. 2). Both ends of the suture were knotted tightly by performing a simple knot. The suture then was looped over a post on the adapter of the materials testing machine to maintain an equal force on both suture limbs. There was no initial tension applied at this point.

Each tendon was pretensioned to $100 \mathrm{~N}$ at a rate of 100 $\mathrm{mm}$ per minute for three cycles to simulate the surgeon's effort to remove slack from the suture-tendon construct in a clinical setting. After pretensioning, each tendon was preloaded to $50 \mathrm{~N}$ for 1 minute followed by cyclic loading. The tendons were loaded for 200 cycles between $50 \mathrm{~N}$ and $200 \mathrm{~N}$ at $200 \mathrm{~mm}$ per minute. These parameters were set

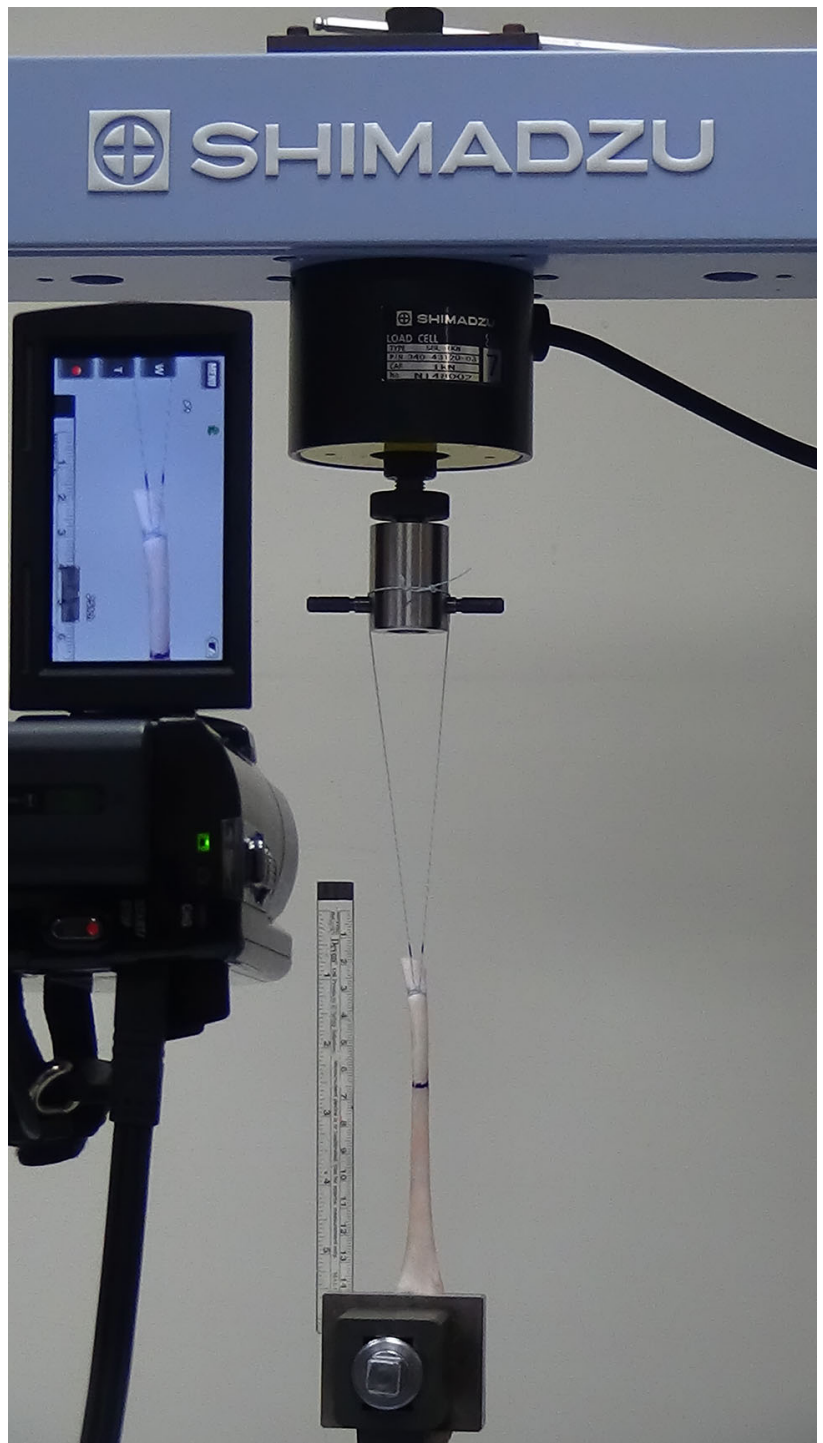

Fig. 2 The biomechanical testing setup in the materials testing system is shown. The suture-tendon construct was secured in a sinusoid clamp. The ends of the sutures were knotted tightly; the suture was looped over a post on the adapter of the materials testing machine. A calibration scale was set next to the suture-tendon construct. A black line was marked $5 \mathrm{~cm}$ from the distal end of the tendon; two black dots were marked on each suture where it extended from the tendon. Video digitizing software and image analysis software then were used to measure elongations of the suture-tendon constructs. 
based on previous studies $[3,10,15,16]$. The $50-\mathrm{N}$ load was selected to simulate the tension on the sutures tied over a cortical bone bridge in ligament-reconstruction procedures [14], while the 200-N cyclic load was chosen because it locates in the ranges of force on the ACL during level walking $(169 \mathrm{~N})$ [5] and the load on the ulnar collateral ligament during throwing $(290 \mathrm{~N})$ [4]. A black line was drawn on the tendon at a point $5 \mathrm{~cm}$ from the distal end of the tendon, and black marks were made on both suture limbs where they extended from the tendon.

Elongation measurements of the suture-tendon constructs for the pretension test were performed by measuring the relative change in distance between the black marks on the sutures and the black line on the tendon. The elongation of both sides of the suture strand was then averaged. After measuring the total elongation of each suture strand after cyclic loading and pretensioning, the changes in elongation of the constructs during the cyclic loading test could be calculated. Percent elongation, calculated as the total elongation change divided by the baseline measurement, was used to normalize the results. A video digitizing system (HDR-XR 269 digital video camera; Sony, Tokyo, Japan) and image analysis software (SigmaScan Pro 5.0) were used to record changes in the markers during testing and measure elongation with digitized markers. An independent observer (SYC) blinded to the application of the suture fixation techniques was assigned to perform all measurements. After cyclic loading, each specimen was loaded to failure in displacement control at a rate of $20 \mathrm{~mm}$ per minute. The displacements and loadings were recorded at a sampling rate of $1 \mathrm{kHz}$. Ultimate failure load was recorded when the maximum tensile force suddenly decreased or discontinued in the load-displacement curve. The mode of failure (eg, suture breakage, suture slippage, or tendon breakage) also was recorded.

\section{Statistical Analysis}

The sample size was calculated according to the elongation after cyclic loading in a pilot study we performed in which there were a total of nine specimens randomly assigned to three groups (rolling hitch, modified rolling hitch, and Krackow stitch). An $\alpha$ equal to 0.05 and a power $(1-\beta)$ of 0.80 were given for this a priori model of power analysis, the calculated effect size of which was 0.60 . The result of the pilot study suggested that if the elongation differed between groups by $60 \%$ of the SD, the study would have $80 \%$ power to identify that difference. A sample size of 10 specimens for each group was determined with the use of G*Power, Version 3.1.3 (available at http://www.gpower.hhu.de; Heinrich Heine-University of Dusseldorf, Dusseldorf, Germany).

Statistical comparisons were conducted with SPSS for Windows, Version 16.0 (SPSS Inc, Chicago, IL, USA), with descriptive statistics, including mean and SD, obtained for each group. The Kruskal-Wallis test with Bonferroni post hoc test was used to compare the crosssectional area, elongation after cyclic loading, and failure load among the different suture configurations. Statistical significance was set as $\mathrm{p}$ less than 0.05 .

\section{Results}

With the numbers available, elongation after cyclic loading was not different among the five successful rolling hitch fixations $(19 \%[1.19 \mathrm{~cm} / 6.17 \mathrm{~cm}] \pm 6 \%)$, the modified rolling hitch fixations $(19 \%[1.11 \mathrm{~cm} / 5.93 \mathrm{~cm}] \pm 6 \%)$, and Krackow stitch fixations $(26 \%[1.41 \mathrm{~cm} / 5.43 \mathrm{~cm}] \pm 6 \%$; p $=0.080)$ (Table 1). In addition, the failure loads of the five successful rolling hitch fixations $(316 \pm 35 \mathrm{~N})$, all modified rolling hitch fixations $(342 \pm 14 \mathrm{~N})$, and all Krackow stitches $(327 \pm 33 \mathrm{~N})$ were not different with the numbers available $(\mathrm{p}=0.18)$.

\section{Discussion}

The rolling hitch and modified rolling hitch are considered to be reliable knotting techniques in ligament-reconstruction procedures [7, 8]. Although some biomechanical studies have suggested the modified rolling hitch to be effective in holding tendon grafts $[2,10]$, to our knowledge

Table 1. Biomechanical properties of the anchor hitch, round turn and two half hitches, rolling hitch, modified rolling hitch, and Krackow stitch

\begin{tabular}{|c|c|c|c|}
\hline Techniques for tendon graft fixation & $\begin{array}{l}\text { Elongation after cyclic } \\
\text { loading }(\% \pm \mathrm{SD})\end{array}$ & $\begin{array}{l}\text { Ultimate failure } \\
\text { load }(N \pm S D)\end{array}$ & Failure mode \\
\hline Rolling hitch & $19 \% \pm 6 \%$ & $316 \pm 35 \mathrm{~N}$ & $\begin{array}{l}\text { Suture slippage }(5 / 10) \text { and rupture } \\
\text { of suture at knot }(5 / 10)\end{array}$ \\
\hline Modified rolling hitch & $19 \% \pm 6 \%$ & $342 \pm 14 \mathrm{~N}$ & Rupture of suture at knot \\
\hline Krackow stitch & $26 \% \pm 6 \%$ & $327 \pm 33 \mathrm{~N}$ & Rupture of suture at knot \\
\hline p Value & 0.08 & 0.18 & \\
\hline
\end{tabular}


no study has compared the traditional suture techniques with the modified rolling hitch or the rolling hitch. We biomechanically evaluated the rolling hitch, modified rolling hitch, and Krackow stitch for preparing tendon grafts. The results of this in vitro porcine biomechanical study suggested that the rolling hitch may not be a good alternative for a suture-tendon construct because half of the rolling hitch fixations failed during cyclic loading. In contrast, all modified rolling hitch fixations held fast during cyclic loading, and elongation after cyclic loading and failure load were not different from the Krackow stitch; therefore, the modified rolling hitch could be considered a potentially viable needleless technique for grasping tendon grafts.

As with all experimental research, this study had certain limitations. First, porcine flexor profundus tendons were used for the experiment, rather than human tendons. Domnick et al. [5] reported that porcine flexor tendons had similar stiffness and type I collagen concentrations compared with human cadaveric semitendinosus tendons, although failure loads were higher, they proposed that porcine flexor tendons are a reasonable surrogate for human semitendinosus tendons. Second, because a single type of suture material was used in this study, no information regarding the potential change in the mechanical behavior of the different grasping suture techniques with different types of suture material can be provided. Third, we evaluated the tensile properties by using a cyclic loading test in a single axial direction; however, this does not capture all physiologic loading conditions. Fourth, although there was only one experienced surgeon performing all suture fixations in our study, it is possible that some variability occurred with respect to the consistency of the suture fixation. Fifth, although the Prusik knot [12] and Wittstein suture loop [17] are also needleless suture techniques in ligament reconstruction, the current study did not evaluate them because a previous study [10] showed that these two knots and the modified rolling hitch technique have similar biomechanical properties in a porcine biomechanical model. Accordingly, since the materials and parameters we used were comparable to those used in the previous study [10], we decided to include the modified rolling hitch only. Sixth, this in vitro biomechanical study did not accurately simulate the intraarticular environment since the testing was not performed in a fluid medium, and we assessed the tensile properties using a cyclic loading test in a single axial direction and at a single rate $(200 \mathrm{~mm} /$ minute), which differs from physiologic conditions. Seventh, we did not specifically assess the graft movement in the clamp, although we did not observe any event of graft slippage or graft movement from the clamp. Eighth, we used black lines on the tendons and sutures rather than markers as indicators for measurement. However, we believe our measurements were reliable, because the distance between the black lines could be measured precisely with an image-analysis system. Finally, we could not determine whether the constricting effects of the needleless techniques would decrease blood flow to the revascularizing graft. Therefore, future studies are needed.

The rolling hitch was proposed as a successful technique in securing autologous hamstring graft in ACL surgery [7]. Highcock et al. [7] believed that using the rolling hitch had several advantages, including that it was timesaving and easy to perform, and there was a smaller circumferential radius of the resultant graft-suture complex. To our knowledge, our study was the first to evaluate the biomechanical properties of the rolling hitch. The results revealed that although the rolling hitch fixation had similar elongation after cyclic loading and similar failure load as the Krackow stitch fixation, it had a 50\% suture slippage rate during cyclic loading, which is the simulation of repetitive force after surgery. The occurrence of suture slippage during cyclic loading may suggest the possibility of graft loosening after surgery before the process of tendon-tobone attachment was completed. The modified rolling hitch was proposed by Hong et al. [8] for tendon graft fixation. In a biomechanical study, Hong et al. [10] proposed the modified rolling hitch to be an attractive technique for suture-tendon constructs because it had similar elongation after cyclic loading and load to failure as the modified Prusik knot and Wittstein suture loop; additionally, Krappinger et al. [12] found that the modified Prusik knot had smaller force-induced displacements than the whipstitch technique. However, whereas Camarda et al. [2] reported that the modified rolling hitch provided better biomechanical properties than the modified finger-trap technique, Su et al. [15] reported that the modified finger-trap featured superior biomechanical properties to the Krackow stitch. Although two prior studies [2,10] did not biomechanically compare the modified rolling hitch and the commonly used needled techniques directly, according to the above previous findings, we could infer that the modified rolling hitch may have comparable or better biomechanical properties compared with the whipstitch or Krackow stitch. In the current study, we biomechanically compared the modified rolling hitch and the Krackow stitch in an in vitro porcine model, and our results suggested that the modified rolling hitch appears to be an attractive technique for a suturetendon construct because it had compatible suture-tendon elongation and failure loads with the Krackow stitch.

Although the results from different biomechanical studies could not be compared directly, the experimental results in our study were consistent with those of previous studies $[9,10,15]$. In the current study, we found $19 \% \pm$ $6 \%$ elongation after cyclic loading and $342 \pm 14 \mathrm{~N}$ failure load in the modified rolling hitch fixation, and the results 
were similar to those in the study by Hong et al. [10] in which they reported $21 \% \pm 10 \%$ elongation and $341 \pm 14$ $\mathrm{N}$ failure load. Su et al. [15] reported 36\% $\pm 5 \%$ elongation after cyclic loading and $381 \pm 25 \mathrm{~N}$ load to failure with the Krackow stitch, whereas Hong et al. [9] reported that the Krackow stitch with five suture throws had an elongation of $26 \% \pm 6 \%$ and failure load of $330 \pm 36 \mathrm{~N}$. In the current study, we reported $26 \% \pm 6 \%$ elongation after cyclic loading and $327 \pm 33 \mathrm{~N}$ failure load with the Krackow stitch fixation.

Several needleless grasping techniques for holding tendon grafts, including the modified Prusik knot [12], Wittstein suture loop [17], rolling hitch [7], and modified rolling hitch [8], have been described. The common characteristic of these knots is that they constrict more tightly when suture limbs are pulled [10]. This characteristic renders these knots suitable for grasping tendons because the diameter of the tendon graft becomes smaller with increasing pulling strength. Regarding the needleless knots in the current study, only the rolling hitch and the modified rolling hitch had a similar effect. Although the rolling hitch had a constricting effect, suture slippage was a concern for this technique.

The rolling hitch, modified rolling hitch, and Krackow stitch techniques had comparable elongation after cyclic loading and load to failure in this in vitro porcine tendon biomechanical evaluation. As this study was a porcine biomechanical study, using cadaveric tendon grafts as the experimental materials could be considered in future studies. In addition, creating an intraarticular environment in which biomechanical testing is performed in the presence of synovial fluid might be worth considering to better simulate the actual conditions in synovial joints. Future histologic analysis in an animal model could further confirm whether the constricting effects of the needleless techniques have biological effects on decreasing blood flow to the revascularizing graft.

Acknowledgments We thank Shing-Yun Chang BS, MSc (Department of Orthopaedic Surgery, National Cheng Kung University Hospital, College of Medicine, National Cheng Kung University, Tainan, Taiwan), for assistance with this project.

\section{References}

1. Becker R, Schroder M, Ropke M, Starke C, Nebelung W. Structural properties of sutures used in anchoring multistranded hamstrings in anterior cruciate ligament reconstruction: a biomechanical study. Arthroscopy. 1999;15:297-300.
2. Camarda L, Pitarresi G, Fazzari F, Tumino D, D'Arienzo M. Biomechanical comparison between the modified rolling-hitch and the modified finger-trap suture techniques. Arch Orthop Trauma Surg. 2016;136:1595-1600.

3. Deramo DM, White KL, Parks BG, Hinton RY. Krackow locking stitch versus nonlocking premanufactured loop stitch for softtissue fixation: a biomechanical study. Arthroscopy. 2008;24:599-603.

4. Dodson CC, Thomas A, Dines JS, Nho SJ, Williams RJ 3rd, Altchek DW. Medial ulnar collateral ligament reconstruction of the elbow in throwing athletes. Am J Sports Med. 2006;34:19261932.

5. Domnick C, Wieskötter B, Raschke MJ, Schulze M, Kronenberg D, Wefelmeier M, Langer MF, Herbort M. Evaluation of biomechanical properties: are porcine flexor tendons and bovine extensor tendons eligible surrogates for human tendons in in vitro studies? Arch Orthop Trauma Surg. 2016;136: 1465-1471.

6. Harvey A, Thomas NP, Amis AA. Fixation of the graft in reconstruction of the anterior cruciate ligament. J Bone Joint Surg Br. 2005;87:593-603.

7. Highcock AJ, Banim R. The rolling hitch surgical knot: an alternative to the Krackow whipstitch for hamstring graft in anterior cruciate ligament reconstruction surgery. Tech Orthop. 2014;29:175-178.

8. 8. Hong CK, Chang CH, Chiang CH, Jou IM, Su WR. Hamstring graft preparation using a modified rolling hitch technique. Arthrosc Tech. 2014;3:e321-324.

9. Hong CK, Lin CL, Chang CH, Jou IM, Su WR. Effect of the number of suture throws on the biomechanical characteristics of the suture-tendon construct. Arthroscopy. 2014;30:1609-1615.

10. Hong CK, Yeh ML, Jou IM, Lin CL, Chang CH, Su WR. Evaluation of 3 needleless grasping suture techniques for softtissue graft fixation: a porcine biomechanical study. Arthroscopy. 2015;31:1151-1155.

11. Krackow KA, Thomas SC, Jones LC. A new stitch for ligamenttendon fixation: brief note. J Bone Joint Surg Am. 1986;68:764766.

12. Krappinger D, Kralinger FS, El Attal R, Hackl W, Haid C. Modified Prusik knot versus whipstitch technique for soft tissue fixation in anterior cruciate ligament reconstruction: a biomechanical analysis. Knee Surg Sports Traumatol Arthrosc. 2007; 15:418-423.

13. McKeon BP, Heming JF, Fulkerson J, Langeland R. The Krackow stitch: a biomechanical evaluation of changing the number of loops versus the number of sutures. Arthroscopy. 2006;22:33-37.

14. Sakaguchi K, Tachibana Y, Oda H. Biomechanical properties of porcine flexor tendon fixation with varying throws and stitch methods. Am J Sports Med. 2012;40:1641-1645.

15. Su WR, Chu CH, Lin CL, Lin CJ, Jou IM, Chang CW. The modified finger-trap suture technique: a biomechanical comparison of a novel suture technique for graft fixation. Arthroscopy. 2012;28:702-710.

16. White KL, Camire LM, Parks BG, Corey WS, Hinton RY. Krackow locking stitch versus locking premanufactured loop stitch for soft-tissue fixation: a biomechanical study. Arthroscopy. 2010;26:1662-1666.

17. Wittstein J, Wilson B, Garrett WE, Toth A. Hamstring graft preparation using a needleless suture loop. J Surg Orthop Adv. 2011;20:142-144. 